\title{
Massive Hemopneumothorax during Breast Augmentation
}

\author{
Seong Yeon Kim, Ji Sung Lee, \\ Young Chul Suh, Young Jin Kim, \\ Jung Ho Lee
}

Department of Plastic and Reconstructive Surgery, Bucheon St. Mary's Hospital, College of Medicine, The Catholic University of Korea, Bucheon, Korea

No potential conflict of interest relevant to this article was reported.

\begin{abstract}
Although it is very rare, hemopneumothorax can occur during breast augmentation. This potentially fatal surgical complication can be experienced by any plastic surgeon who performs breast augmentation surgery. In this article, we present a case from our institution and review the related literature in order to explore the etiological mechanism of hemopneumothorax, as well as preventive measures and treatments. In addition, we have tried to emphasize the importance of a thorough knowledge of anatomical variations and proper surgical techniques as ways to minimize the likelihood of this complication.
\end{abstract}

Keywords Breast, Hemorrhage, Hemothorax, Pneumothorax, Wounds and injuries

\section{INTRODUCTION}

Hematoma and infection are the most common complications of breast augmentation; however, cases of pneumothorax and hemothorax are rarely reported $[1,2]$. However, according to Osborn and Stevenson [1], one-third of the members of the American Society of Plastic Surgery who performed breast augmentation had experienced these complications at least once, and one-tenth had experienced them twice or more, which shows that they are more common than generally considered. Pneumothorax and hemothorax should be appropriately managed because they can cause life-threatening consequences, such as tamponade [3].

The mechanism of hemopneumothorax after breast augmentation is the spontaneous intraoperative rupture of an underlying bleb or bulla or retrocostal bleeding due to an injury of the intercostal perforator artery or fascia [4]. It may also be caused by direct intraoperative trauma to the pleura or the lung, an injury to the lung parenchyma with a needle during a local injection, and high

Received: Jul 11, 2018 Revised: Jul 16, 2018 Accepted: Jul 17, 2018 Correspondence: Jung Ho Lee Department of Plastic and Reconstructive Surgery, Bucheon St. Mary's Hospital, College of Medicine, The Catholic University of Korea, 327beon-gil, Sosa-ro, Wonmi-gu, Bucheon 14647, Korea. E-mail: tfm0822@catholic.ac.kr

Copyright @ 2018 The Korean Society for Aesthetic Plastic Surgery.

This is an Open Access article distributed under the terms of the Creative Commons Attribution Non-Commercial License (http://creativecommons.org/licenses/by-nc/4.0/) which permits unrestricted non-commercial use, distribution, and reproduction in any medium, provided the original work is properly cited. www.e-aaps.org anesthetic ventilation pressure during general anesthesia [1].

We experienced a case of hemopneumothorax in a patient who underwent submuscular breast augmentation with an inframammary fold (IMF) incision. In this article, we aimed to determine the pathogenic mechanism and perioperative precautions suitable for preventing hemopneumothorax from an anatomical perspective and to emphasize the preventive measures and treatments for this fatal complication based on a literature review.

\section{CASE REPORT}

A 45-year-old woman who underwent breast augmentation via the IMF approach at a local clinic was transferred to our hospital for hemopneumothorax. On physical examination, there was an incision measuring approximately $5 \mathrm{~cm}$ along the left IMF, and a $2 \times 4$ $\mathrm{cm}$ intercostal muscle and fascial defect was observed in the sixth intercostal space. In addition, an area of parietal pleura was exposed through the defect (Fig. 1). On computed tomography (CT), massive hemopneumothorax on the left side with soft tissue emphysema was observed (Fig. 2).

Emergency hemostasis and chest tube drainage (CTD) insertion were performed by the cardiothoracic surgical team, and reconstruction of the intercostal fascial defect was planned. Intraoperatively, suture materials and coagulation wounds caused by electrocautery were observed around the parietal pleura and endothoracic fascia, presumably because multiple attempts at bleeding control and sutures for defect coverage were made at the local clinic. The fascial defect was covered with a prosthesis (Gore-Tex) by the car- 


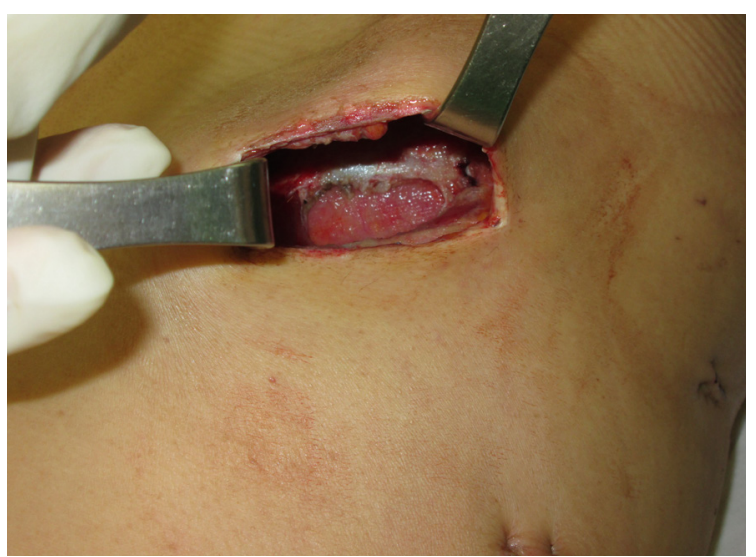

Fig. 1. An intercostal fascial defect measuring $2 \times 4 \mathrm{~cm}$ was observed through the left inframammary fold incision, and the parietal pleura was exposed through the defect.

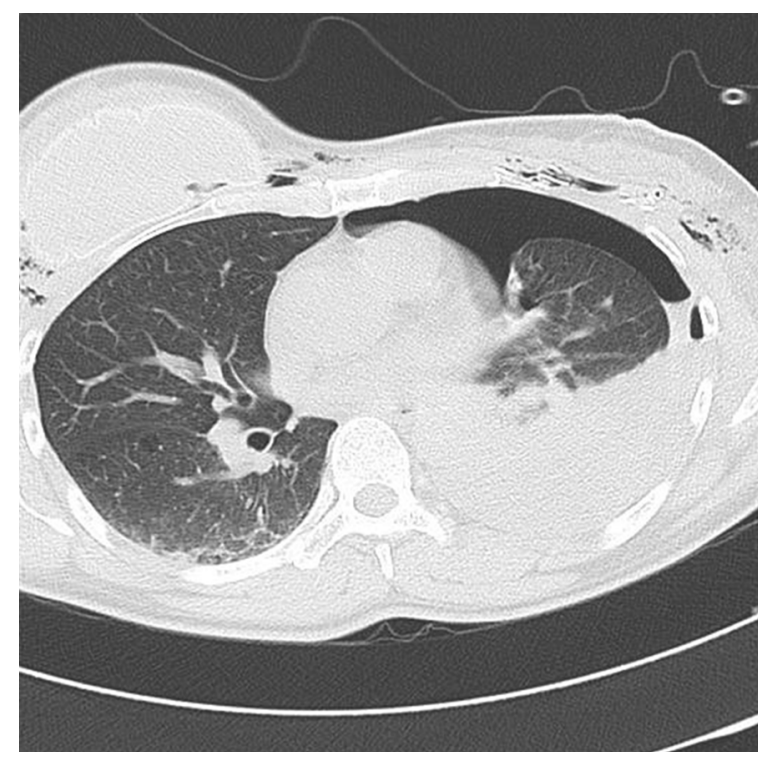

Fig. 2. Computed tomography confirmed massive left-sided hemothorax and pneumothorax.

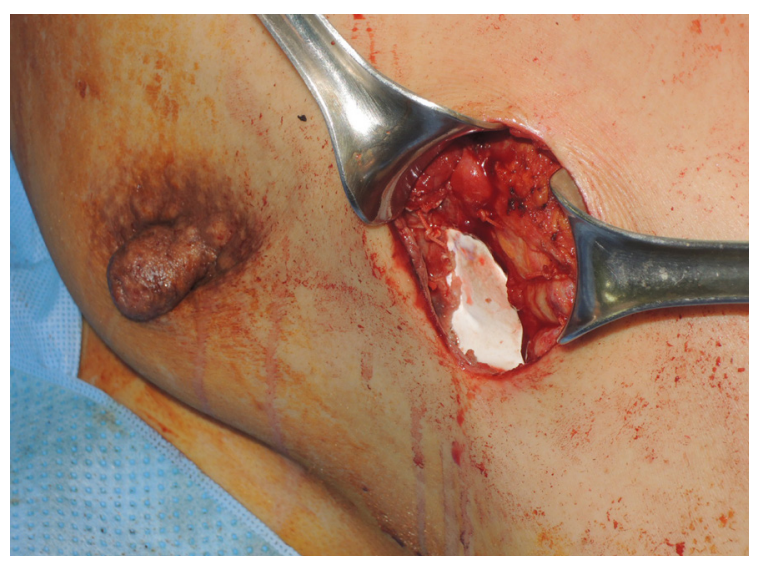

Fig. 3. A prosthesis (Gore-Tex) was applied to the fascial defect.
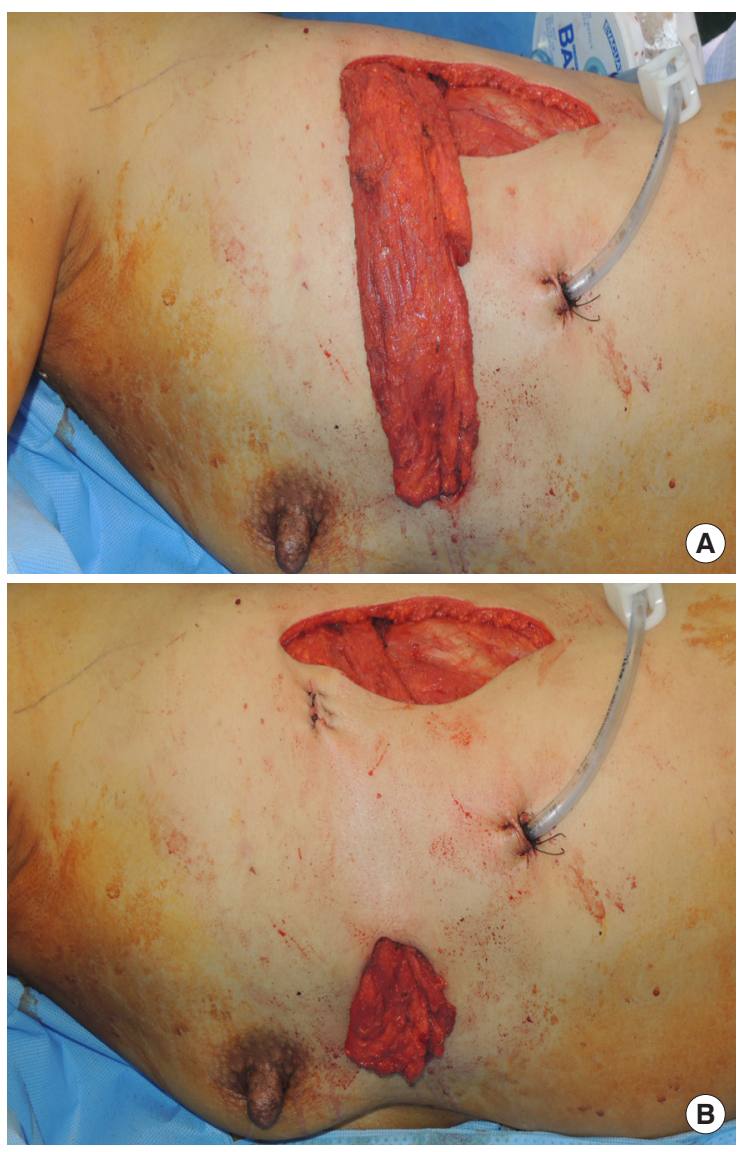

Fig. 4. (A) Elevation of split latissimus dorsi muscle flap based on the descending branch of thoracodorsal vessel. (B) Transposition of the muscle flap over the defect.

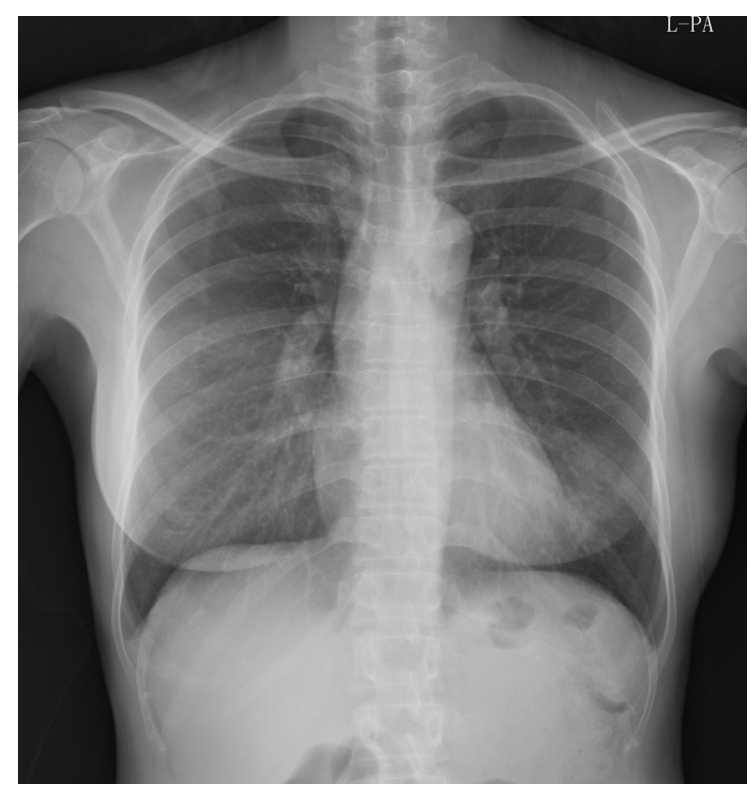

Fig. 5. Follow-up chest X-ray 1 month postoperatively. No abnormalities, such as hemopneumothorax, were found. 

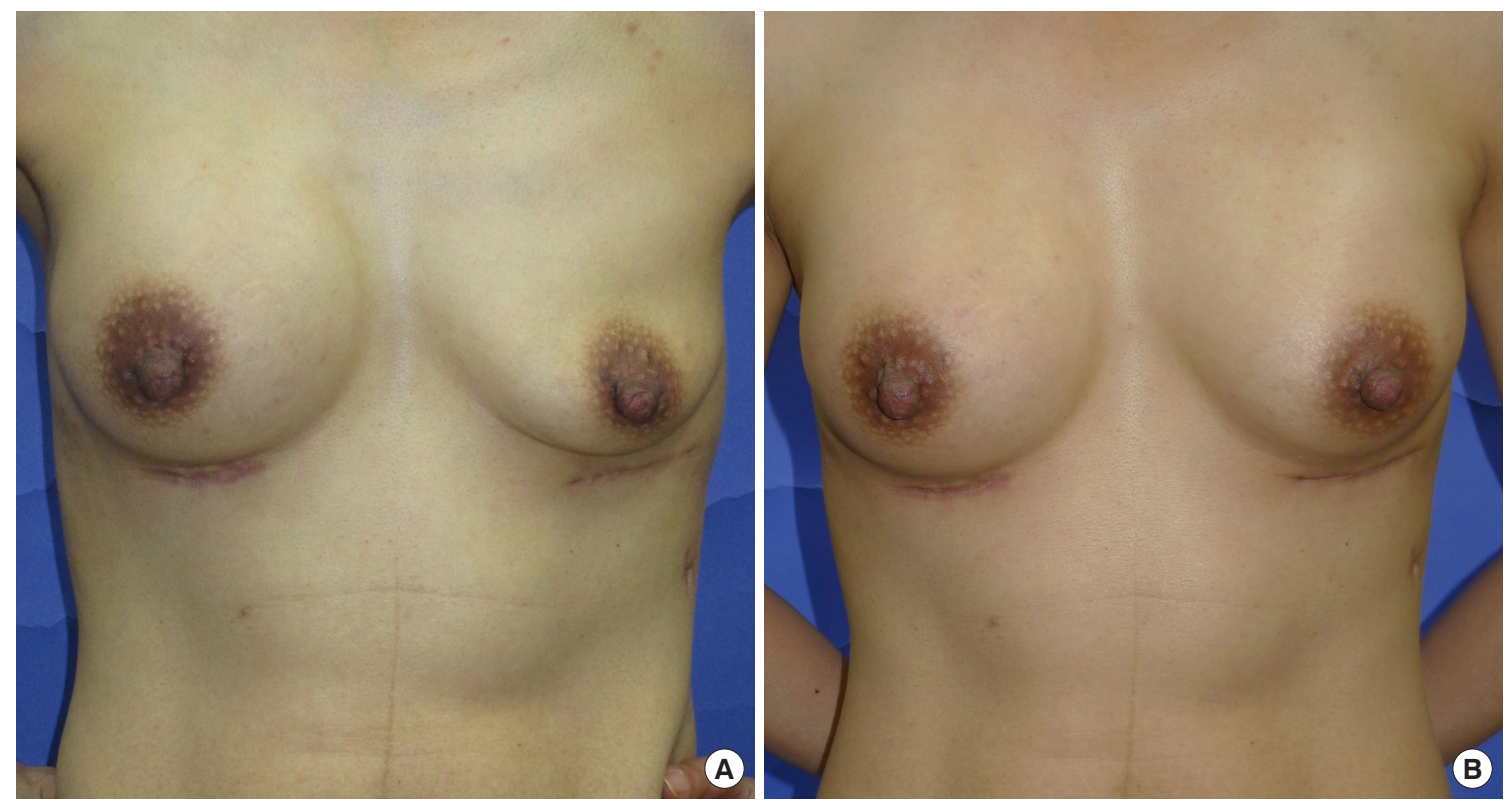

Fig. 6. (A) Complete wound healing without complications at 2 months postoperatively. (B) Left-sided breast augmentation using a dual-plane pocket and $200-\mathrm{mL}$ silicone implants was performed to achieve symmetry of both breasts.

diothoracic surgical team, and then we transposed a latissimus dorsi muscle flap over the prosthesis (Fig. 3 and 4).

A follow-up chest X-ray at 1 month postoperatively did not show abnormal findings (Fig. 5). After complete healing without further complications in 2 months, left-sided breast augmentation was successfully performed through the previous IMF incision (Fig. 6).

\section{DISCUSSION}

When performing submuscular breast augmentation, intercostal muscle injury can occur due to incorrect recognition of the surgical plane. Because the intercostal muscle is very thin, and its injury is closely associated with hemopneumothorax, surgeons must be very familiar with the anatomical position and variants of the pectoralis major muscle to prevent intercostal muscle injury.

The inferior border of the costal origin of the pectoralis major muscle arises in the sixth rib in $68 \%$ of individuals, in the seventh rib in $20 \%$, and in the fifth rib in $12 \%$, and the levels of origin of the left and right pectoralis major are different in $16 \%$ of cases. The level of the costal origin of the pectoralis major and the IMF level were the same in $61 \%$ of cases. However, the muscle origin has been reported to be 1 rib level higher than the IMF level in 36\% of cases [5]. Moreover, the costal origin of the pectoralis minor muscle is also variable. According to a report by Anson et al. [6] that analyzed 100 cadaveric specimens, the costal origin of the pectoralis minor muscle was connected from the second to fifth ribs in $42 \%$ of cases, from the third to fifth ribs in $28.5 \%$ of cases, from the second to fourth ribs in $15 \%$ of cases, and from the third to fourth ribs in $5 \%$ of cases [7]. Therefore, these anatomical differences must be considered during the IMF approach, and the dissection plane must be predicted when creating a submuscular pocket in order to identify the surgical plane accurately.

Another factor that complicates surgery, aside from anatomical variants of the pectoralis muscle, is intraoperative bleeding. During submuscular augmentation, intercostal perforators are found in the area of dissection. If bleeding occurs due to injury to these intercostal perforators, hemostasis is difficult due to the narrow operating window. In addition, careless hemostasis by electrocautery may cause intercostal muscle tearing or intercostal fascial injury. Therefore, the surgeon must be familiar with and aware of the courses of these perforators and perform meticulous bleeding control in order to prevent fascial injury [8].

The intercostal perforator is divided into internal, medial, and lateral segments, and the internal and medial segments are especially likely to be affected during submuscular dissection. In a CT analysis of 30 women with 164 perforators, 52 perforators were internal, 37 were medial, and 75 were lateral. The internal perforators were located, on average, $2.57 \mathrm{~cm}$ from the xiphoid process on the $\mathrm{X}$-axis and $1.42 \mathrm{~cm}$ on the $\mathrm{Y}$-axis, while the medial perforators were located, on average, $8.59 \mathrm{~cm}$ from the xiphoid process on the $\mathrm{X}$-axis and $0.82 \mathrm{~cm}$ on the Y-axis. The lateral perforators were located, on average, $13.37 \mathrm{~cm}$ from the xiphoid process on the $\mathrm{X}$-axis and $-0.95 \mathrm{~cm}$ on the Y-axis [8]. Therefore, knowing this information before the operation can help predict the location of perforators during submuscular dissection, enabling the surgeon to perform a safe dissection. Furthermore, according to a cadaveric study by Gil- 
lis et al. [9], the cutaneous perforator branches from the internal mammary artery at $7.0 \pm 1.4 \mathrm{~mm}$ away from the sternal margin. The cutaneous perforator of the internal thoracic artery has been reported to course, on average, along the second to fifth intercostal spaces at $20 \mathrm{~mm}$ farther from the midsternal line, which indicates that the areas within $20 \mathrm{~mm}$ surrounding the midsternal line in the second and third intercostal spaces and within $30 \mathrm{~mm}$ surrounding the midsternal line in the fourth intercostal space are designated as danger zones. In other words, dissection within these areas requires great attention. In particular, the perforator tends to be larger in the second and third intercostal spaces, and severe bleeding may occur if an injury is sustained in these areas [10].

In our case, considering the location of the intercostal fascial defect, it is thought that the medial intercostal perforator in the sixth intercostal space ruptured during submuscular dissection and that the intercostal muscle and fascia defect may have occurred due to excessive and repeated attempts to perform hemostasis. Furthermore, repairing the intercostal defect with suture material probably aggravated the situation. Therefore, the location of perforators along the dissection plane must be considered and verified before surgery, and caution must be taken to prevent iatrogenic injury to the fascia through meticulous bleeding control.

If a small fascial laceration is discovered intraoperatively, a catheter must first be inserted. Then, the fascia must be repaired using a purse-string or interrupted suture, and the lungs must be expanded through positive pressure ventilation before removing the catheter [1]. If pneumothorax or hemothorax occurs due to pleural injury, as in our case, the pleural defect must be fundamentally repaired. However, the defect may become larger if the thin pleura is repaired with excessive sutures. Therefore, the pleura should be repaired with polyglycolic acid mesh or topical sealant, after which a CTD should be inserted [11]. If a fascial injury is not found intraoperatively, but the patient presents with dyspnea or chest discomfort postoperatively, oxygen saturation must be evaluated and a chest X-ray performed. If minimal pneumothorax occurs, conservative inpatient care with $\mathrm{O}_{2}$ supplementation should be considered. CTD insertion or a surgical procedure may be required for severe cases.

To prevent postoperative hemopneumothorax, accurate dissection of the submuscular pocket is mandatory. Additionally, the anatomical position of the main perforators that can cause bleeding during dissection must be considered and meticulous hemostasis should be performed to prevent iatrogenic intercostal muscle injury. Finally, informed consent must be obtained from the patient regarding the possibility of pneumothorax or hemothorax as a complication of surgery, and for the timely treatment of unrecognized complications, the patient should be educated to visit the hospital immediately if dyspnea occurs postoperatively.

\section{PATIENT CONSENT}

The patient provided written consent for the use of her images.

\section{REFERENCES}

1. Osborn JM, Stevenson TR. Pneumothorax as a complication of breast augmentation. Plast Reconstr Surg 2005;116:1122-6; discussion 7-8.

2. Keyes GR, Singer R, Iverson RE, et al. Analysis of outpatient surgery center safety using an internet-based quality improvement and peer review program. Plast Reconstr Surg 2004;113:1760-70.

3. Nagrani T, Neuman T, Baldari D, et al. Tamponade following breast augmentation. Ann Plast Surg 2010;64:141-3.

4. Domergue S, Ziade M, Falinower H, et al. Hemothorax in a patient undergoing breast augmentation surgery. Plast Reconstr Surg 2015; 136:124e-5e.

5. Madsen RJ Jr, Chim J, Ang B, et al. Variance in the origin of the pectoralis major muscle: implications for implant-based breast reconstruction. Ann Plast Surg 2015;74:111-3.

6. Anson BJ, Beaton LE, McDonald JJ. The origin of the M. pectoralis minor. J Anat 1938;72:629-30.

7. Sanchez ER, Sanchez R, Moliver C. Anatomic relationship of the pectoralis major and minor muscles: a cadaveric study. Aesthet Surg J 2014;34:258-63.

8. Carrasco-López C, Julian Ibañez JF, Vilà J, et al. The anterior intercostal artery flap: anatomical and radiologic study. Plast Reconstr Surg 2017;139:613e-9e.

9. Gillis JA, Prasad V, Morris SF. Three-dimensional analysis of the internal mammary artery perforator flap. Plast Reconstr Surg 2011;128: 419e-26e.

10. Li S, Liu C, Mu D, et al. What is the optimal range of medial pocket dissection in breast augmentation? An anatomical study of Chinese women. Aesthetic Plast Surg 2016;40:863-8.

11. Van Schil PE, Hendriks JM, Lauwers P. Pleural tears: are all holes the same? Eur J Cardiothorac Surg 2009;35:41-2. 